\title{
Procrastination is Detrimental to Undergraduate Students' Self-Rated Creativity: The Mediating Role of State Anxiety*
}

Jia-Wei Lim

Universiti Tunku Abdul Rahman, Malaysia

Joo-Yee Phang

Universiti Tunku Abdul Rahman, Malaysia

\author{
Mei-Yan Low \\ Universiti Tunku Abdul Rahman, Malaysia
}

\author{
Chee-Seng Tan \\ Universiti Tunku Abdul Rahman, Malaysia \\ E-mail address: tcseng@utar.edu.my
}

ARTICLE INFO

\section{Keywords:}

Procrastination

Creativity

State Anxiety

Mediation Analysis

Malaysia

\section{A B STRACT}

Procrastination has been found to negatively impact academic and job performance. However, little attention has been given to the effect of procrastination on creative performance. Moreover, past studies have mainly focused on the antecedent role of anxiety in procrastination. The impact of procrastination on anxiety is not clear, though literature has suggested that procrastination may induce anxiety. The primary question addressed in the present study is whether and how procrastination influences creativity. Some exceptional studies have indicated that procrastination allows incubation and hence is conducive to creativity. However, on the basis of the literature, we argued and hypothesized that procrastination may impair creativity through state anxiety. A total of 218 Malaysian undergraduate students were recruited via social networking sites and responded to a packet of online survey questionairres, including self-report of procrastination tendency, state and trait anxiety, and creativity. Results showed that procrastination was positively associated with state and trait anxiety and negatively correlated with creativity. A negative relationship was also observed between state and trait anxiety and creativity. In addition, mediation analysis supported our hypothesis that state anxiety mediates the relationship between procrastination and creativity after controlling for the effect of trait anxiety. Specifically, people tend to experience feeling anxious when they postpone task completion. The high level of anxiety, in turn, is negatively associated with creative performance. As a whole, the findings not only offer the first empirical evidence supportive of the detrimental effect of procrastination on creativity, but also reveal the underlying process. Future directions and limitations are also explored. 
Have you ever delayed work until the following day or even to just before the deadline? Procrastination is a voluntary delay in an intended course of action, though a worse outcome is only to be expected (Steel, 2007). Sadly, procrastination is ubiquitous in students as well as in the general population.

Over the years, procrastination has been found to have a negative effect on academic performance (Kim \& Seo, 2015; Owens, 1997; You, 2015), job satisfaction (Mohsin \& Ayub, 2014), mental health (Fritzsche, Rapp Young, \& Hickson, 2003; Mohsin \& Ayub, 2014; Rice, Richardson, \& Clark, 2012) and self-efficacy (Klassen, Krawchuk, \& Rajani, 2008). Using a longitudinal design, Patrzek, Sattler, van Veen, Grunschel, and Fries (2014) examined responses of between 1359 and 2207 university students collected in two waves and found that academic procrastination affected them in different ways and led to academic misconduct, such as using fraudulent excuses, plagiarism and fabrication or falsification of data.

The impact of procrastination on creativity has also been documented. Some researchers found that procrastination is positively associated with creativity (e.g., DíazMorales, Cohen, \& Ferrari, 2008; Subotnik, Steiner, \& Chakraborty, 1999). Specifically, procrastination allows individuals to have more time for incubation. Setting the task aside for a while is said to promote remote association of ideas and hence, individuals are more likely to generate novel responses (e.g., Hao, Liu, Ku, Hu, \& Runco, 2015). On the contrary, literature on anxiety suggests that procrastination could be harmful to creativity (e.g., Byron \& Khazanchi, 2011). Procrastinators tend to experience anxiety whereby high anxiety levels are negatively associated with creativity (Byron \& Khazanchi, 2011). As a result, it is reasonable to believe that procrastination may impair creativity. Little attention, however, has been given to the hypothetical negative effect of procrastination on creativity.

Thus, the present study aims to extend the literature by examining the impact of procrastination on creativity and the mechanism underlying this relationship. In the following section, we first reviewed the relationship between procrastination and creativity. Then, we discussed the hypothetical mediating role of anxiety in the procrastination-creativity linkage by reviewing the impact of procrastination on anxiety and the relationship between anxiety and creativity.

\section{LITERATURE REVIEW}

\section{Procrastination and Creativity}

Procrastination refers to the intention to delay the completion or the commencement of a given task (Brownlow \& Reasinger, 2000; Solomon \& Rothblum, 1984). Procrastination has been found to have a negative impact on performance and mental health. Little has been said about the relationship between procrastination and creativity. 
Some exceptional studies, however, have suggested a positive relationship between procrastination and creativity (Díaz-Morales et al., 2008; Subotnik et al., 1999; Van Eerde, Beeftink, \& Rutte, 2015). Subotnik et al. (1999), for instance, interviewed 19 individuals who won the Westinghouse Science Talent Search in 1983 to investigate the prevalence of procrastination in different domains, such as routine work, health and creative expression. It was found that among the 8 respondents employed in creativityrelated positions, half of those who rated themselves as elite in their field reported that they used procrastination for incubation to reach the best solutions to questions they were examining. The result suggests that procrastination is conducive to creative performance, as individuals had used the time to scrutinize available solutions and, finally, selected the ideal one. This was further documented by Gevers, Mohammed, and Baytalskaya (2015) who suggested that procrastination might be an incubation period for creativity. In addition, Díaz-Morales et al. (2008) revealed that the beneficial influence of procrastination on creativity could be due to the preference for imaginative thinking style. Diaz-Morales and colleagues examined the influence of motivational, cognitive and behavioural personality styles on avoidant procrastination - postponement of a task's completion, when individuals perceive that they lack competence to complete the task. The analysis of 1027 adults' self-reports showed that highly avoidant procrastinators scored higher in the imaginative scale of thinking style than low avoidant procrastinators. The findings suggest that avoidant procrastinators' tendency to use imaginative thinking styles may result in more creative performance.

Although studies have found that procrastination is conducive to creative performance, it is often argued that procrastination could be harmful to creativity for two reasons. First, research has consistently found procrastination to have a negative impact on academic performance. As academic performance is positively associated with creativity (e.g., McCabe, 1991), it is believed that procrastination is detrimental to creative performance. In addition, the literature on anxiety suggests that procrastination may paradoxically impair creativity. Specifically, studies have documented that procrastination is positively correlated with anxiety (Haycock, McCarthy, \& Skay, 1998; van Eerde, 2003), with anxious people tending to have low performance in divergent thinking (Byron \& Khazanchi, 2011), an index that has been widely used in creativity research. Therefore, it seems sensible to assume that procrastination is indirectly harmful to creative performance due to anxiety.

\section{The Mediating Role of Anxiety}

Procrastination and anxiety. Anxiety refers to an unpleasant emotion associated with fear (Clark \& Beck, 2012). Anxiety can be further conceptualized into state anxiety and 
trait anxiety (Spielberger, 1976). Trait anxiety is a stable disposition to experience anxiety. Individuals with high trait anxiety experience intensive anxiety more frequently than those who have low trait anxiety. State anxiety, on the other hand, is a transient, unpleasant emotional response that one experiences when coping with threatening circumstances.

All along, anxiety has been consistently found to have a positive relationship with procrastination (Fritzsche et al., 2003; Haycock et al., 1998; van Eerde, 2003). For example, Fritzsche and colleagues (2003) found that undergraduate students with a high tendency to procrastinate on writing tasks reported a high level of general anxiety (trait anxiety) besides state anxiety in writing papers. Similarly, Onwuegbuzie (2004) and Dunn (2014) found a positive relationship between procrastination and statistics anxiety. Glick and Orsillo (2015), on the other hand, indicated that students are likely to develop academic procrastination when threatened by negative thoughts (e.g., I am an incompetent writer) and feelings (e.g., anxiety). The findings suggest that procrastination could be one of the means for individuals to avoid negative emotions, like fear, anxiety and self-evaluation which emerged from the aversive tasks (Glick, Millstein, \& Orsillo, 2014; Sirois \& Pychyl, 2013).

Although most studies suggest that anxiety is the antecedent to procrastination, we argue that the relationship between anxiety and procrastination could be bidirectional. In other words, while anxiety may lead to procrastination, it is possible that procrastination may result in anxiety. Procrastinators may experience unpleasant feelings (e.g., anxiety, stress) when the due date is around the corner but the task is yet to be completed. For instance, Rothblum, Solomon, and Murakami (1986) and Ferrari (1991) found that high procrastinators reported higher levels of anxiety than their counterparts. Notably, the effect of procrastination on anxiety has been given considerably little attention thus far.

As such, some explanations have lent their support to the effect of procrastination on anxiety. For example, failure in completing tasks on time is troublesome and the negative consequence of late submission may elicit depression and anxiety (Beswick, Rothblum, \& Mann, 1988). Indeed, people tend to feel guilty, anxious and depressed when they are unable to cope with time pressure (Ferrari \& Dovidion, 2001). Moreover, procrastinators may engage in other alternative activities (e.g., hanging out with friends) that can elicit the feeling of pleasure in order to avoid or suppress these negative feelings. The attempt to elicit pleasant thoughts lends support to the bidirectional effect of anxiety on procrastination. After all, individuals do not have to participate in other activities to boost their mood if being able to procrastinate can reduce the negative feelings induced by anxiety. Taken together, anxiety (e.g., fear of failure) increases the tendency to procrastinate. The postponement and failure of completion, in turn, increase the level of anxiety. 
Anxiety and Creativity. Creativity has been conceptualized as the ability to change idea, product, and problem-solving into new domains (Csikszentmihalyi, 1996). Although the conceptualization of creativity varies from one field to another, researchers generally agree that creativity consists of two components: originality and usefulness (Runco \& Jaeger, 2012). In other words, a creative product should be novel and is able to solve a problem encountered by people in the field.

Meanwhile, the literature suggests that anxiety could be detrimental to creative performance as the anxious mindset inhibits novelty-seeking behaviour which interferes with the creative process (Eysenck, Derakshan, Santos, \& Calvo, 2007; Fales et al., 2008; George \& Zhou, 2007; Heilman, 2014). According to the Cognitive Interference Theory (Sarason, 1988), individuals who experience anxiety tend to think of, or recall unfavorable experiences. The focus on undesirable experiences restricts the individual's retrieval from long-term memory causing limited accessibility of information and eventually decreases creative performance (Heilman, Nadeau, \& Beversdorf, 2003; Mumford \& Gustafson, 1988). Furthermore, Cognitive Interference Theory also suggests that anxiety may narrow attention at the conceptual level and reduce working memory (Fales et al., 2008). The deficit in working memory may restrict individuals to combine ideas and to create mental images which in turn decrease creativity (Scott, Lonergan, \& Mumford, 2005).

Empirical studies have found that anxiety is negatively associated with creativity (Byron \& Khazanchi, 2011; Friedman \& Förster, 2008). Findings for emotion and creativity indeed indicate that negative emotion such as anxiety and fear are harmful to creativity (e.g., Baas, De Dreu, \& Nijstad, 2008). Byron and Khazanchi (2011) discovered that anxiety is negatively associated with creative performance. They meta-analyzed 59 independent studies that investigated the relationship between anxiety and creativity. The results support the notion that anxiety has a negative impact on creativity. It has also been found that trait anxiety has a stronger negative relationship with creative performance than state anxiety does. In addition, anxiety exerts a greater detrimental effect on creative performance when the creativity tasks are complex.

In addition, de Acedo-Baquedano and de Acedo-Lizarraga (2012) administered the State-Trait inventory and Test of Creative Imagination to 89 participants between the ages of 12 and 14 . They found that both trait and state anxiety were negatively correlated with verbal and graphic creativity. Silvia and Kimbrel (2010) examined the relationship among dimensions of depression, anxiety and social anxiety and several aspects of creativity (including divergent thinking, creative self-concepts, everyday creative behaviours and public creative achievements) using latent variable models. 
They found that anxiety and depression symptoms have an impact on creativity, though the effects ranged from small to insignificant. The findings suggest that anxiety is harmful to creative performance.

\section{The Present Study}

The primary objective of the present study was to investigate the impact of procrastination on creativity. Although a few studies have found a positive relationship between procrastination and creativity, we argue that procrastination is harmful to creative performance because the anxious feeling elicited by delaying tasks may narrow one's attention and inhibit novelty-seeking behaviors.

The second objective was to examine the effect of procrastination on anxiety. Procrastination has been consistently found to have a positive relationship with anxiety. Most of the studies have focused on the antecedent role of anxiety in procrastination. Although some literature suggest that procrastinators tend to experience anxiety when they postpone the completion of a task, little attention has been given to a hypothetical relationship of this kind. Ruling out the relatively stable characteristics of trait anxiety that might not be easily influenced by other constructs, state anxiety, rather than trait anxiety, was tested in the present study. On the basis of the literature, we also proposed and tested a mediation model to illustrate the underlying mechanism for the procrastination - creativity linkage. Specifically, we postulated that procrastination has an indirect effect on creative performance through anxiety.

\section{METHODOLOGY}

\section{Participants}

A total of 218 undergraduate students (73 males, 145 females) from local and private universities in Malaysia voluntarily participated in the study. The participants were aged between 19 to 25 years $(M=21.99, S D=1.35)$ and were recruited through convenience sampling and snowball sampling. The sample comprised 204 Chinese, 12 Indians, and 2 Malays.

\section{Instruments}

Tuckman Procrastination Scale (Tuckman, 1991). The Tuckman Procrastination Scale consists of 16 items that assess the procrastination tendencies of college students. Respondents indicated the extent to which the items depict them on a 4-point Likert scale, ranging from 1 (That's me for sure) to 4 (That's not me for sure). Initially, four items (Items 7, 12, $14 \& 16$ ) required reverse scoring and a lower total score represented higher procrastination. However, for ease of interpretation, we reverse scored all the items so that higher scores indicated greater procrastination tendency. The scale was found to have good internal consistency (Cronbach $\alpha=.83$ ). 
State-Trait Inventory for Cognitive and Somatic Anxiety (STICSA; Ree, French, MacLeod, \& Locke, 2008). Participants responded to the 21 items on a 4-point Likert scale, ranging from 1 (Not at all) to 4 (Very much so). The STICSA comprised two subscales: cognitive anxiety (10 items) and somatic anxiety (11 items). Sample items for the cognitive anxiety and somatic anxiety subscales are "I think that the worst will happen" and "My breathing is fast and shallow" respectively. Moreover, with different instructions, the STICSA may assess both state (the feeling of respondents right now, at the present moment) and trait anxiety (the feeling of respondents in general) of a person. The STICSA was found to have excellent internal consistency $(\alpha=.91, .88, .82$ for trait anxiety, trait cognitive, \& trait somatic subscales; $\alpha=.95, .92, .91$ for state anxiety, state cognitive, \& state somatic subscales) and validity (Gros, Antony, Simms, \& McCabe, 2007; Gros, Simms, \& Antony, 2010).

Self-perceived Creativity (Zhou \& George, 2001). The 13-item self-report measure was initially developed for supervisors to evaluate their subordinates' creativity. However, some researchers (e.g., Reiter-Palmon, Morral, Kaufman, \& Santo, 2012; Tan, Lau, \& Lee, 2017; Tan, Lau, Kung, \& Kailsan, 2016) modified items in the scale (e.g., "the employee comes up with new and practical ideas to improve performance" to "I come up with new and practical ideas to improve performance") and used it to measure selfperceptions of creativity, which is consistent with the interest of the present study on selfperception. Participants answered the items on a 5-point Likert scale, ranging from 1 (not characteristics at all) to 5 (very characteristics), to indicate the extent to which the creative behaviors are part of their characteristics. The measure showed a high internal consistency in the sample $(\alpha=.92)$.

\section{Procedure}

An online survey was created using Qualtrics. The first three researchers posted the online survey link on their social networking sites. Additionally, the researchers also sent the link to undergraduates at different universities in Malaysia via email. Respondents in both conditions were asked to invite their friends to participate in the study through sharing of the link. After giving their informed consent, participants were asked to fill in demographic information and answer the questionnaires on procrastination, anxiety and creativity.

\section{RESULTS}

Table 1 shows the results of the intercorrelation, mean, standard deviation, skewness, and kurtosis for the variables in the present study. Normality is assumed as the absolute z scores for skewness were below 3.29 (Kim, 2013) except for the state anxiety and state somatic subscale. The issue was resolved by log transforming the data. As the results 
obtained from the transformed scores were comparable to the results from original scores, we reported the latter for ease of interpretation.

Overall, correlation analyses showed that all variables were significantly related to one another. Procrastination was positively associated with all anxiety scores and was negatively associated with self-perceived creativity. On the other hand, self-perceived creativity was found to have a negative relationship with all anxiety scores except trait somatic anxiety. Generally, the results provide preliminary support for our hypotheses. The state somatic and cognitive subscales, as well as the trait somatic and cognitive subscales, were highly correlated. We then used the overall state and trait anxiety scores, rather than the scores from the four subscales, for further analysis.

Table 1

Descriptive Statistics and Intercorrelations for Variables $(\mathbf{N}=\mathbf{2 1 8})$

\begin{tabular}{|c|c|c|c|c|c|c|c|c|}
\hline Variable & 1 & 2 & $2 a$ & $2 b$ & 3 & $3 a$ & $3 b$ & 4 \\
\hline 1. Procrastination ${ }^{a}$ & 1 & & & & & & & \\
\hline 2. State Anxiety & .27 & 1 & & & & & & \\
\hline 2a. State Somatic & $.18^{\star *}$ & .94 & 1 & & & & & \\
\hline 2b. State Cognitive & .33 & .94 & .77 & 1 & & & & \\
\hline 3. Trait Anxiety & .32 & .76 & .68 & .76 & 1 & & & \\
\hline 3a. Trait Somatic & .25 & .72 & .72 & .64 & .92 & 1 & & \\
\hline 3b. Trait Cognitive & .33 & .70 & .55 & .76 & .94 & .72 & 1 & \\
\hline 4. Creativity & $-.19^{\star \star}$ & $-.20^{\star *}$ & $-.15^{\star}$ & $-.23^{\star \star}$ & $-.15^{\star}$ & -.13 & $-.14^{\star}$ & 1 \\
\hline$M$ & 38.95 & 1.67 & 1.53 & 1.82 & 1.90 & 1.76 & 2.06 & 3.37 \\
\hline$S D$ & 7.03 & 0.57 & 0.57 & 0.64 & 0.50 & 0.48 & 0.61 & 0.59 \\
\hline Skewness & 0.05 & 0.83 & 1.20 & 0.47 & 0.42 & 0.56 & 0.34 & -0.27 \\
\hline Kurtosis & -0.17 & -0.11 & 0.52 & -0.50 & -0.59 & -0.29 & -0.52 & 1.41 \\
\hline
\end{tabular}

Note. $S E_{\text {skewness }}=0.17, S E_{\text {kurtosis }}=0.33,{ }^{*} p<.05,{ }^{* \star} p<.01$, coefficients in bold were significant at .001 level. ${ }^{a}$ procrastination was reverse-scored such that a higher score indicates a higher tendency to procrastinate.

We hypothesized that state anxiety could mediate the relationship between procrastination and creativity. The SPSS macro PROCESS model 4 (Hayes, 2013) with 10,000 bootstrapping sample was used to examine the hypothesized indirect effect. The indirect effect is considered statistically significant if the bias-corrected $95 \%$ confidence interval (Cl) does not contain zero.

Mediation analysis on creativity (with procrastination as predictor, state anxiety as mediators, and trait anxiety as covariate) showed that procrastination was positively associated with state anxiety, $B=0.02$, $S E=0.005, t=4.14, p<.001$. State anxiety, but not trait anxiety, was negatively associated with creativity, $B=-0.22$, $S E=0.11, t=2.02, p=.04$. The total effect of procrastination on creativity was sta- 
tistically significant $(B=-0.01, S E=0.006, t=2.34, p=.02)$. The direct effect of procrastination on creativity remained significant $(B=-0.01, S E=0.006, t=2.27$, $p=.02$ ), after controlling the effects of state and trait anxiety. More importantly, the indirect effect of procrastination on creativity via state anxiety was found significant, $B=-0.005, S E=0.003,95 \% \mathrm{Cl}[-0.01,-0.0004]$ (see Figure 1).

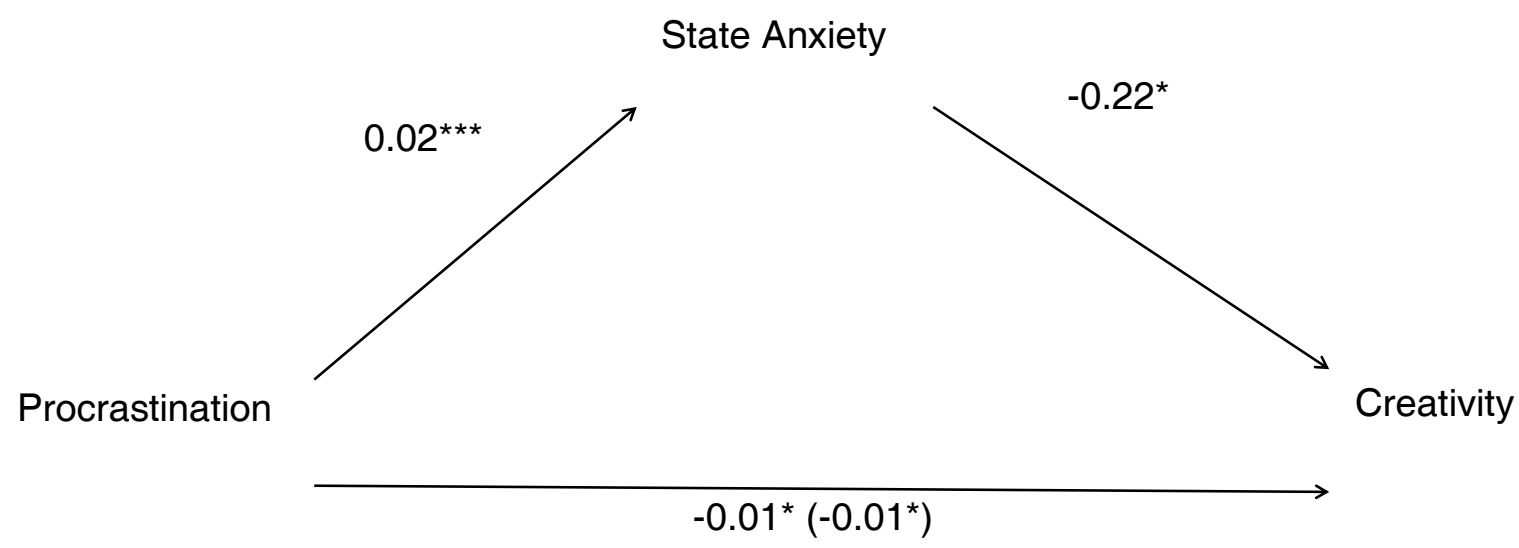

Note. ${ }^{\star} p<.05,{ }^{* \star \star} p<.001$

Figure 1. State anxiety mediates the effect of procrastination on creativity while controlling the effect of trait anxiety. Procrastination was reverse-scored with a higher score indicating a higher tendency to procrastinate. $N=218$.Values shown are unstandardized coefficients. The total effect is shown in parenthesis.

Overall, the results support the view that state anxiety mediates the relationship between procrastination and creativity. As the Tuckman Procrastination Scale was reverse scored with a higher score indicating a higher tendency to procrastinate, the negative coefficient of the indirect effect implies that people with high procrastination tend to have low creativity. In particular, people are likely to experience state anxiety when they procrastinate. The high anxiety level is thus associated with low creative performance.

\section{DISCUSSION}

Does procrastination enhance or impair creativity? The findings of the present study favoured the detrimental approach. People who have a tendency to procrastinate tend to report low creativity. Moreover, this negative relationship is mediated by state anxiety. As such, the present study is the first to offer insight into the underlying mechanism for the impact of procrastination on creativity. Specifically, our findings indicate that procrastinators may experience anxiety when they delay tasks. The anxious feelings, in turn, hinder creative performance. The results also suggest the necessity to consider the role of anxiety and other negative feelings when examining the effect of procrastination.

Prior to the study, we hypothesized a negative relationship between procrastination and creativity. As expected, our results showed the negative influence of procrastination 
on creativity. Our findings thus provide the first empirical evidence supportive of the detrimental effect of procrastination on creativity. Note that our results contradict previous studies that report procrastination as being positively related to creativity (e.g., DíazMorales et al., 2008; Subotnik et al., 1999). One possible reason for the contrast lies in the differences in thinking styles. Previous studies indicate that Western participants tend to use a creative thinking style rather than a concrete thinking style in completing tasks (Díaz-Morales et al., 2008). Asians, on the other hand, are prone to use a concrete thinking style instead of a creative thinking style (Wong, 2004). Moreover, it has been found that a creative thinking style (e.g., imaginative thinking) is conducive to creative performance (Díaz-Morales et al., 2008). For instance, in the distant future time perspective condition, participants who were assigned the task of generating abstract solutions were more creative than their counterparts who generated concrete solutions (Förster, Friedman, \& Liberman, 2004). Therefore, it is reasonable to assume that the effect of procrastination on creativity may depend on an individual's thinking style. For example, procrastinators who adopt the creative thinking style are more likely to generate unique and useful solutions in reducing anxiety and completing the task than those who adopt the concrete thinking style. Future studies are thus recommended to examine the role of thinking style in the relationship between procrastination and creativity.

Notably, our data suggest that people may experience negative feelings such as anxiety when they delay work. Such anxious feelings limit their scope of attention, prohibit novelty-seeking behaviours and then decrease their creative performance. However, it is important to note that we are not saying that people are unable to generate creative products when facing deadlines. In fact, we acknowledge that last minute work could be creative if individuals used the time wisely in exploring potential options and scrutinizing the best solution. In other words, the influence of procrastination on creative performance may depend on the way an individual utilizes the time. Taken together, our findings suggest that it is not sufficient to simply focus on procrastination behaviour. Future research on procrastination should consider the intention of procrastination, that is, whether postponement is used as a means to search for a better solution or to escape from aversive tasks.

Earlier literature has suggested that one of the explanations for the discrepancies in the relationship between procrastination and creativity is the mental preparation of procrastinators. Procrastinators who use their time for incubating ideas and searching for better solutions (e.g., Subotnik et al., 1999) would mentally engage themselves in their tasks. On the contrary, because of fears of failure, judgment and success, avoidant procrastinators would tend to escape from aversive tasks and not engage themselves in their tasks 
both mentally and physically (Ferrari, O'Callaghan, \& Newbegin, 2005). Compared to avoidant procrastinators, procrastinators who mentally engage themselves in tasks would be expected to be more mentally well-prepared. Moreover, it has been documented that mental preparation can reduce the level of anxiety in different fields, such as sport (e.g., Ryska, 1998), operations (e.g., Protogerou et al., 2015), and psychotherapy (Deane, Spicer, \& Leathem, 1992). This might also be true in the context of procrastination. In other words, procrastinators who are mentally well-prepared for tasks are more likely to have a lower level of anxiety than avoidant procrastinators. As anxiety has a negative impact on creativity (e.g., Byron \& Khazanchi, 2011; Heilman, 2014), the level of mental preparation could be an important factor in determining the creativity level of the procrastinators.

Consistent with previous studies (e.g., Byron \& Khazanchi, 2011; de AcedoBaquedano \& de Acedo-Lizarraga, 2012), both trait and state anxiety are found to be negatively related to creativity in the present study. However, only state anxiety would negatively influence self-rated creativity. The results not only lend further support to the detrimental effect of state anxiety on creativity, but also suggest that state anxiety and trait anxiety may play a different role in creativity. Future studies are recommended to further examine the different effects of trait and state anxiety in the relationship between procrastination and creativity. For instance, it is assumed that individuals with high trait anxiety tend to procrastinate. The delay of work is then associated with state anxiety which is harmful to creativity.

Overall, the results pertaining to procrastination and anxiety have replicated and extended previous research, showing that anxiety is one of the factors in procrastination. Our results have shown that anxiety could be the effect of procrastination. In other words, people who procrastinate are likely to experience anxious feelings. We hypothesize that the effect could be due to procrastination, which restricts the development of trait selfefficacy. People with low self-efficacy tend to think that they are unable to cope with the threatening situation, which in turn increases their anxiety level. Empirical studies are therefore needed to examine the hypothetical role of self-efficacy. It is also important to further examine the bidirectional relationship between procrastination and anxiety. Although our results support the notion that anxiety is an effect of procrastination, past studies have shown compelling evidence that anxiety is the antecedent of procrastination. In other words, it is possible that procrastination mediates the relationship between anxiety and creativity. It is therefore suggested that future studies should employ an experimental design to clarify the mediating role of procrastination and anxiety. 


\section{Limitations and Suggestions}

There are some limitations to the present study. First, although the study moves beyond examining general anxiety which was the focus of previous studies (e.g., Silvia \& Kimbrel, 2010) and examined the role of state anxiety, the effect of trait anxiety was not directly examined. It is not clear whether trait anxiety also plays a role in the relationship between procrastination and creativity. Based on the literature (e.g., Fritzsche et al., 2003), it is hypothesized that anxiety has a loop effect on procrastination. Specifically, people with a tendency to react negatively to challenging situations (e.g., high trait anxiety) are inclined to procrastinate in order to avoid the negative emotions elicited by aversive tasks. The procrastination behaviour, as our findings show, in turn, increases state anxiety. It would then be interesting to examine this assumption in future studies to further clarify the relationship between anxiety and procrastination. In addition, the present study focused on selfperceived creativity and hence provides little information with regard to the impact of procrastination on actual creative performance. It is also noteworthy that different types of procrastination have been suggested (e.g., decisional procrastination, avoidant procrastination). As such, future research may consider replicating the present study with other types of procrastination to examine the effect on creative performance.

Data collection was conducted online via social networking sites in the present study. Compared to the paper-and-pencil method, online surveys have enabled us to reach out to a broader range of participants. Nevertheless, the online method is not without its limitations. The online setting does not allow participants to seek clarification if any is needed. We are unable to rule out the possibility that participants may have misunderstood some items, though we reviewed the items prior to data collection. Furthermore, the convenience and snowball sampling method limited the generalization of the findings. Our sample consisted of mainly Chinese students and hence, it is not clear if the results can be extended to other ethnicity groups.

Finally, it is important to emphasize that it is not possible to draw the conclusion of a causal link between variables from mediation analysis findings, though the results support the possibility of such a link. Future researchers are strongly recommended to carry out experiments to replicate the findings of the present study by examining the potential causal relationships involved.

In conclusion, the present study offers new input for procrastination research, showing evidence for the detrimental effect of procrastination on young adults' creativity. We also provide evidence that procrastination can be the cause, rather than the effect of anxiety. More importantly, our results reveal the underlying mechanism for the negative 
impact of procrastination on creativity. In particular, the anxious feelings due to task postponement may limit cognitive flexibility and impair one's creative performance. As creativity has been recognized as one of the important abilities in order to achieve success, it is necessary that more attention is given to teaching undergraduate students how to reduce procrastination and anxiety.

\section{REFERENCES}

Baas, M., De Dreu, C., K. W., \& Nijstad, B. A. (2008). A meta-analysis of 25 years of mood-creativity research: Hedonic tone, activatioin, or regulatory focus? Psychological Bulletin, 134(6), 779-806.

Beswick, G., Rothblum, E. D., \& Mann, L. (1988). Psychological antecedents of student procrastination. Australian Psychologist, 23, 203-217.

Brownlow, S., \& Reasinger, R. D. (2000). Putting off until tomorrow what is better done today: Academic procrastination as a function of motivation toward college work. Journal of Social Behavior \& Personality, 15, 15-34.

Byron, K., \& Khazanchi, S. (2011). A meta-analytic investigation of the relationship of state and trait anxiety to performance on figural and verbal creative tasks. Personality and Social Psychology Bulletin, 37(2), 269-283. doi: 10.1177/0146167210392788

Clark, D. A., \& Beck, A. T. (2012). The anxiety and worry workbook: The cognitive behavioral solution. New York, NY: The Guilford Press.

Csikszentmihalyi, M. (1996). Creativity : flow and the psychology of discovery and invention: New York, NY: Harper Perennial.

De Acedo-Baquedano, M. T. S., \& de Acedo-Lizarraga, M. L. S. (2012). Relationships between state and trait anxiety with verbal and graphic creativity in students in compulsory Secondary Education. Electronic Journal of Research in Educational Psychology, 10(3), 1123-1138.

Deane, F. P., Spicer, J., \& Leathem, J. (1992). Effects of videotaped preparation information on expectations, anxiety, and psychotherapy outcome. Journal of Consulting and Clinical Psychology, 60(6), 980-984.

Díaz-Morales, J. F., Cohen, J. R., \& Ferrari, J. R. (2008). An integrated view of personality styles related to avoidant procrastination. Personality and Individual Differences, 45(6), 554-558. doi: http://dx.doi.org/10.1016/j.paid.2008.06.018

Dunn, K. (2014). Why wait? The influence of academic self-regulation, intrinsic motivation, and statistics anxiety on procrastination in online statistics. Innovative Higher Education, 39(1), 33-44.

Eysenck, M. W., Derakshan, N., Santos, R., \& Calvo, M. G. (2007). Anxiety and cognitive performance: Attentional control theory. Emotion (Washington, D.C.), 7(2), 336-353. 
Fales, C. L., Barch, D. M., Braver, T. S., Burgess, G. C., Schaefer, A., Mennin, D. S., \& Gray, J. R. (2008). Anxiety and cognitive efficiency: Differential modulation of transient and sustained neural activity during a working memory task. Cognitive, Affective and Behavioral Neuroscience, 8(3), 239-253. doi: 10.3758/CABN.8.3.239

Ferrari, J. R. (1991). Compulsive procrastination: Some self-reported characteristics. Psychological Reports, 68(2), 455-458.

Ferrari, J. R., \& Dovidion, J. F. (2001). Behavioral information search by indecisives. Personality and Individual Differences, 30, 1113-1123.

Ferrari, J. R., O'Callaghan, J., \& Newbegin, I. (2005). Prevalance of the procrastination in the United States, United Kingdom, and Australia: Arousal and avoidance delays among adults. North American Journal of Psychology, 7(1), 1-6.

Förster, J., Friedman, R. S., \& Liberman, N. (2004). Temporal construal effects on abstract and concrete thinking: Consequences for insight and creative cognition. Journal of Personality and Social Psychology, 87(2), 177-189.

Friedman, R. S., \& Förster, J. (2008). Activation and measurement of motivational states. Handbook of approach and avoidance motivation (pp. 235-248). New York, NY: Psychology Press.

Fritzsche, B. A., Rapp Young, B., \& Hickson, K. C. (2003). Individual differences in academic procrastination tendency and writing success. Personality and Individual Differences, 35, 1549-1557. doi: 10.1016/S0191-8869(02)00369-0

George, J. M., \& Zhou, J. (2007). Dual tuning in a supportive context: joint contributions of positive mood, negative mood, and supervisory behaviors to employee creativity. The Academy of Management Journal, 50(3), 605-622.

Gevers, J., Mohammed, S., \& Baytalskaya, N. (2015). The conceptualisation and measurement of pacing styles. Applied Psychology, 64(3), 499-540. doi: 10.1111/ apps.12016

Glick, D. M., Millstein, D. J., \& Orsillo, S. M. (2014). A preliminary investigation of the role of psychological inflexibility in academic procrastination. Journal of Contextual Behavioral Science, 3(2), 81-88.

Gros, D. F., Antony, M. M., Simms, L. J., \& McCabe, R. E. (2007). Psychometric Properties of the State-Trait Inventory for Cognitive and Somatic Anxiety (STICSA): Comparison to the State-Trait Anxiety Inventory (STAI). Psychological Assessment, 19(4), 369-381.

Gros, D. F., Simms, L. J., \& Antony, M. M. (2010). Psychometric properties of the statetrait inventory for cognitive and somatic anxiety (sticsa) in friendship dyads. Behavior Therapy, 41, 277-284. doi: 10.1016/j.beth.2009.07.001 
Hao, N., Liu, M., Ku, Y., Hu, Y., \& Runco, M. A. (2015). Verbal divergent thinking facilitated by a pleasurable incubation interval. Psychology of Aesthetics, Creativity, and the Arts, 9(3), 286-295. doi: 10.1037/a0038851

Haycock, L. A., McCarthy, P., \& Skay, C. L. (1998). Procrastination in college students:

The role of self-efficacy and anxiety. Journal of Counseling and Development, 76, 317-324.

Hayes, A. F. (2013). Introduction to mediation, moderation, and conditional process analysis : A regression-based approach: New York, NY: The Guilford Press.

Heilman, K. M. (2014). Creativity and the brain. New York, NY: Psychology Press.

Heilman, K. M., Nadeau, S. E., \& Beversdorf, D. O. (2003). Creative innovation: Possible brain mechanisms. Neurocase, 9(5), 369-379.

Kim, H.-Y. (2013). Statistical notes for clinical researchers: Assessing normal distribution (2) using skewness and kurtosis. Restorative Dentistry \& Endodontics, 38(1), 52-54. doi: 10.5395/rde.2013.38.1.52

Kim, K. R., \& Seo, E. H. (2015). The relationship between procrastination and academic performance: A meta-analysis. Personality and Individual Differences, 82, 26-33. doi: 10.1016/j.paid.2015.02.038

Klassen, R. M., Krawchuk, L. L., \& Rajani, S. (2008). Academic procrastination of undergraduates: Low self-efficacy to self-regulate predicts higher levels of procrastination. Contemporary Educational Psychology, 33, 915-931. doi: 10.1016/j.cedpsych.2007.07.001

McCabe, M. P. (1991). Influence of creativity and intelligence on academic performance. The Journal of Creative Behavior, 25(2), 116-122. doi: 10.1002/j.2162-6057.1991.tb01361.

Mohsin, F. Z., \& Ayub, N. (2014). The relationship between procrastination, delay of gratification, and job satisfaction among high school teachers. Japanese Psychological Research, 56(3), 224-234. Doi: 10.1111/jpr.12046

Mumford, M. D., \& Gustafson, S. B. (1988). Creativity syndrome: integration, application, and innovation. Psychological Bulletin, 103(1), 27-43.

Onwuegbuzie, A. J. (2004). Academic procrastination and statistics anxiety. Assessment \& Evaluation in Higher Education, 29(1), 3-19.

Owens, A. M. N. I. (1997). Procrastination in high school achievement: A causal structural model. Journal of Social Behavior \& Personality, 12(4), 869-887.

Patrzek, J., Sattler, S., van Veen, F., Grunschel, C., \& Fries, S. (2014). Investigating the effect of academic procrastination on the frequency and variety of academic misconduct: $A$ panel study. Studies in Higher Education, 40(6), 1014-1029. doi: 10.1080/03075079.2013.854765 
Protogerou, C., Fleeman, N., Dwan, K., Richardson, M., Dundar, Y., \& Hagger, M. S. (2015). Moderators of the effect of psychological interventions on depression and anxiety in cardiac surgery patients: a systematic review and meta-analysis. Behavior Research and Therapy, 73, 151-164.

Ree, M. J., French, D., MacLeod, C., \& Locke, V. (2008). Distinguishing cognitive and somatic dimensions of state and trait anxiety: Development and validation of the statetrait inventory for cognitive and somatic anxiety (STICSA). Behavioural and Cognitive Psychotherapy, 36(03), 313-332.

Reiter-Palmon, R., Morral, E., Kaufman, C., \& Santo, J. B. (2012). Evaluation of self-perceptions of creativity: Is it a useful criterion? Creativity Research Journal, 24(2-3), 107-114.

Rice, K. G., Richardson, C. M. E., \& Clark, D. (2012). Perfectionism, procrastination, and psychological distress. Journal of Counseling Psychology, 59(2), 288-302. doi: http://dx.doi.org/10.1037/a0026643

Rothblum, E. D., Solomon, L. J., \& Murakami, J. (1986). Affective, cognitive, and behavioral differences between high and low procrastinators. Journal of Counseling Psychology, 33(4), 387-394.

Runco, M. A., \& Jaeger, G. J. (2012). The standard definition of creativity. Creativity Research Journal, 24(1), 92-96. Doi: 10.1080/10400419.2012.650092

Ryska, T. A. (1998). Cognitive-behavioral strategies and precompetitive anxiety among recreational athletes. The Psychological Record, 48(4), 697-708.

Sarason, I. G. (1988). Anxiety, self-preoccupation and attention. Anxiety Research, 1(1), 3-7. doi: 10.1080/10615808808248215

Scott, G. M., Lonergan, D. C., \& Mumford, M. D. (2005). Conceptual combination: Alternative knowledge structures, alternative heuristics. Creativity Research Journal, 17(1), 79-98. doi: 10.1207/s15326934crj1701_7

Silvia, P. J., \& Kimbrel, N. A. (2010). A dimensional analysis of creativity and mental illness: Do anxiety and depression symptoms predict creative cognition, creative accomplishments, and creative self-concepts? Psychology of Aesthetics, Creativity, and the Arts, 4(1), 2-10. doi: 10.1037/a0016494

Sirois, F., \& Pychyl, T. (2013). Procrastination and the priority of short-term mood regulation: Consequences for future self. Social and Personality Psychology Compass, 7(2), 115-127. doi: 10.1111/spc3.12011

Solomon, L. J., \& Rothblum, E. D. (1984). Academic procrastination: Frequency and cognitive-behavioral correlates. Journal of Counseling Psychology, 31, 503-509. 
Spielberger, C. D. (1976). The nature and measurement of anxiety. In C. D. Spielberger \& R. Diaz-Guerrero (Eds.), Cross-cultural anxiety (pp. 3-10). Washington, DC: Hemisphere.

Steel, P. (2007). The nature of procrastination: A meta-analytic and theoretical review of quintessential self-regulatory failure. Psychological Bulletin, 133(1), 65-94.

Subotnik, R., Steiner, C., \& Chakraborty, B. (1999). Procrastination revisited: The constructive use of delayed response. Creativity Research Journal, 12(2), 151-160.

Tan, C. S., Lau, X. S., \& Lee, L. K. (2017). The mediating role of creative process engagement in the relationship between shyness and self-rated creativity. Journal of Creative Behavior. Advance online publication. doi: 10.1002/jocb.173

Tan, C. S., Lau, X. S., Kung, Y. T., \& Kailsan, R. (2016). Openness to experience enhances creativity: The mediating role of intrinsic motivation and the creative process engagement. Journal of Creative Behavior. Advance online publication. doi: 10.1002/jocb.170

Tuckman, B. W. (1991). The development and concurrent validity of the procrastination scale. Educational \& Psychological Measurement, 51(2), 473.

Van Eerde, W. (2003). A meta-analytically derived nomological network of procrastination. Personality and Individual Differences, 35(6), 1401-1418. doi: http://dx.doi.org/ 10.1016/S0191-8869(02)00358-6

Van Eerde, W., Beeftink, F., \& Rutte, C. G. (2015). Working on something else for a while: Pacing in creative design projects. Time \& Society. doi: 10.1177/0961463 x15577274

Wong, J. K.-K. (2004). Are the learning styles of Asian international students culturally or contextually based? International Education Journal, 4(4), 166.

You, J. W. (2015). Examining the Effect of Academic Procrastination on Achievement Using LMS Data in e-Learning. Journal of Educational Technology \& Society, 18(3), 64-74.

Zhou, J., \& George, J. M. (2001). When job dissatisfaction leads to creativity: Encouraging the expression of voice. Academy of Management Journal, 44(4), 682-696. doi: $10.2307 / 3069410$

Corresponding author at: Chee-Seng Tan, Department of Psychology and Counselling, University Tunku Abdul Rahman, Jalan University, Bandar Barat, 31900 Kampar, Perak, Malaysia.

E-mail: tcseng@utar.edu.my

CCopyright by Faculty of Pedagogy and Psychology, University of Bialystok,

20 Swierkowa St., 15-328 Bialystok, Poland tel. +48857457283 